\title{
Bioética de la anorexia nerviosa ¿autonomía, beneficencia o responsabilidad?
}

\section{G ustavo Figueroa}

\section{Ethics in anorexia nervosa: Autonomy, beneficence or responsibility?}

In patients with anorexia nervosa, ethical dilemmas will appear in case of difficult and serious medical situations. Although the principle of beneficence could be used to argue for the use of coercive medical therapies, the respect for autonomy must take precedence. The latter is a central principle of bioethics. However, the interactions between autonomy, beneficence, non-maleficence, and justice become quite complex when these patients need to be treated. Difficulties must be overcome and a balance between these considerations must be reached. This paper gives an account of ethical values relevant to moral assessment in anorexia nervosa: conviction, responsibility and care (Rev Méd Chile 2008; 136: 659-65).

(Key words: Anorexia nervosa; Health care ethics; Social responsibility)

Recibido el 14 de noviembre, 2007. Aceptado el 3 de marzo, 2008.

Departamento de Psiquiatría, Universidad de Valparaíso. Chile

L a anorexia se ha constituido en una afección de crecimiento explosivo, con elevadas tasas de prevalencia en mujeres adolescentes y jóvenes, catalogándosela de catástrofe nacional antes que de enfermedad catastrófica ${ }^{1-4}$. Los dilemas éticos se han debatido entre respeto irrestricto a la autonomía desde que se alcanza determinada edad ("menor maduro") o aplicación de tratamientos médicos coercitivos por motivos de beneficencia para prevenir secuelas graves 0 aun mortales ${ }^{5,6}$. El presente trabajo intenta mostrar que la dicotomía entre derechos a la "libertad" o a

Correspondencia a: Dr. Gustavo Figueroa. Teléfono: (32) 269367.

E mail: gusta.figueroa@holtmail.com - gufigueroa@vtr.net la "vida" es uno de los conflictos éticos, entre varios, y que decidir conductas terapéuticas complejas sólo desde dos principios puede omitir cuidados, desestimar consecuencias peligrosas 0 fomentar hábitos perjudiciales para la salud.

\section{Procedimientos DE DeCISIÓN}

La anorexia plantea una serie de cuestionamientos morales que culminan en la inminencia de muerte (Tabla 1). Jonsen et al propusieron un procedimiento de toma de decisiones que considera cuatro dimensiones siguendo una secuencia progresiva (Tabla 2) ${ }^{7}$. El primer parámetro incluye a la anorexia dentro de los trastornos CEPA (crónico, eficaz tratamiento, paliativo, ambulatorio), pero el cuadro clínico se puede tornar crítico y requerir hospitalización, alimentación forzada por sonda, 
Tabla 1. D imensiones bioéticas de la anorexia mental

\author{
Autonomía - Beneficencia \\ Pertenencia del cuerpo humano \\ Competencia \\ Calidad de vida \\ Decisiones de sustitución \\ Privacidad \\ Intimidad \\ Confidencialidad \\ Agotamiento del equipo de salud (burnout)
}

vigilancia permanente, apremio, pérdidas de la privacidad, intimidad y confidencialidad. El médico recurre al principio de beneficencia impulsado por la necesidad imperiosa de curar complicaciones peligrosas, y fundamentalmente salvar la vida a la anoréctica, aun cuando ella rechace la ayuda y deba imponérsela contra su voluntad ${ }^{8-12}$.

El segundo parámetro establece inequívocamente que a toda adulta le es inherente poseer capacidades psicológicas que la hacen competente para tomar decisiones y resolver su propia condición según sus convicciones íntimas cuando se le proporciona información comprensible, sustancial y veraz, convicciones que son intransferibles e irrenunciables ${ }^{13-15}$. La anoréctica tiene el privilegio de rechazar o rehusarse a cualquier tratamiento aunque parezca insensato, irrazonable 0 aun contrario a sus intereses, evaluados según criterios del clínico o su familia ${ }^{16-20}$. El consentimiento informado evalúa de manera objetiva su capacidad de autonomía radical -facultad para decidir voluntariamente sin coerción ni manipulación- para vivir de acuerdo a su concepción de existencia digna $\mathrm{y}$, por ello también, como indica el tercer parámetro, para optar por la calidad de vida que le parezca más suya como persona ${ }^{21-23}$.

\section{ÉTICA DE MÁXIMOS Y DE MÍNIMOS}

La ética de máximos comprende la vida privada de la anoréctica que ella escoge y se apropia libremente -la "perfección" a la que aspira-, a diferencia de la de mínimos, que estipula sus obligaciones públicas que pueden exigírsela coactivamente para su cumplimiento -los "deberes" básicos pero ineludibles que debe satisfacer-. Hasta aquí hemos visto que la conducta del médico oscila entre promover el principio prima facie de beneficencia o acatar el de autonomía; al confrontar ambos actúa al interior de la ética de máximos, esto es, de una "responsabilidad fuerte" o de excelencia. Empero desde su nacimiento la bioética ha conferido primacía implícita a la autonomía por sobre la beneficencia, en contra de la tradición médica milenaria; así, prioriza aquellas perfecciones morales a las que la anoréctica, como sujeto personal, tiene derecho a aspirar en conformidad con su voluntad soberana ${ }^{23-27}$. Su consecuencia es crucial: cada acción terapéutica debe justificarse con razones muy poderosas cuando, contrariamente, no se sigue 0 no se obedece el sistema explícito de valores de la paciente (ej: el "privilegio terapéutico" sólo como medida extrema de protección).

Tabla 2. Parámetros para la toma de decisión en la práctica clínica*
1. Las indicaciones médicas
Principios de beneficencia y no maleficencia
2. Las preferencias del paciente
Principio de respeto a la autonomía
3. La calidad de vida
Principios de beneficencia, no maleficencia y respeto a la autonomía
4. Los rasgos contextuales
Principios de justicia y lealtad

*Adaptado de: Jonsen, AR, Siegler, M, Winsdale WJ. Clinical ethics. A practical approach to ethical decisions in clinical medicine. $5^{\text {th }}$ edition. New York: McGraw-Hill, 20027. 
También se procede contra la práctica histórica el impedir al médico que la fuerce activamente a realimentarse para que no muera. Desde Hipócrates imperaba sin contrapeso el precepto de favorecer o "beneficiar" - "la vida". Ahora domina el de "no hacer daño" o no-perjudicar - "la libertad" de JS Mill. Vale decir, nunca se puede hacer el bien o aun salvarle la vida sin el consentimiento de la anoréctica; se puede apelar, inducir o convencer a que la enferma acepte la terapéutica, pero no imponérsela argumentando que se obra así por su beneficio ${ }^{28}$.

Conviene hacer una precisión que suele pasar inadvertida. En bioética se habla de autonomía al referirse a una paciente capaz de y legitimada para tomar decisiones acerca de cuestiones de salud que la afectan vitalmente -es un derecho general. Pues bien, lo que está en juego en la anoréctica es otro aspecto: la autorrealización individual antes que la noción universal de autonomía de la voluntad, según Kant ${ }^{29}$. Son sus ideales privados de propia realización, sus proyectos íntimos frente al de los otros, su idea de vida virtuosa o felicidad. Esto es, "automealización" individual frente a "autonomía" humana. Cuando el médico evalúa la paciente lo hace pensando en su existencia imepetible asumida con responsabilidad, en el "sentido" de sus acciones y decisiones, antes que en las "razones" o "argumentos lógicamente deducidos" - "sentido más pleno" contra "razones correctas"25,26. Sólo esto le autoriza a cuestionar, más aún, dejar en suspenso las convicciones que están sustentando la paciente anoréctica. Considerada desde la perspectiva de la enferma misma, sus creencias parecen expresar "razones" nacidas desde su voluntad, pero su autorrealización individual está ejencida sin reflexión vital genuina -moda, imitación, capricho, oposicionismo-, esto es, asumida sin "sentido" biográfico, vale decir, im-propia. El inconveniente está en que no ha sido posible elaborar instrumentos para objetivar el voluntarismo versus la autenticidad de sus creencias y hábitos.

\section{CONTEXTO CLÍNICO-INDIVIDUAL}

Esta antinomia entre principios permite comprender los graves tropiezos para obtener que la elección de una conducta médica sea éticamente justificada. Además en el cuadro anoréctico las circunstancias son ambiguas y las consecuencias imprevisibles.

1. Ser menor de edad constituye una "incapacidad temporal" que requiere que las decisiones sean tomadas por sus familiares o quienes ejercen la patria potestad. Recientemente se postula la existencia del "menor maduro", que pone en entredicho las doctrinas tradicionales del desarrollo de la conciencia moral. Frente a cada anoréctica se necesita evaluar rigurosamente su competencia para tomar decisiones desde que se sitúa en edades superiores a los 12 años ${ }^{30,31}$.

2. Los cambios orgánicos en la anorexia acostumbran alterar profundamente la capacidad de decidir según la razón. Esta capacidad, antes que un problema de "umbral", es un "continuum". Debe examinarse mediante pruebas y, en especial, repetidamente en el tiempo, junto con explorar la naturaleza de las situaciones que influyen y matizan los rendimientos (Tabla 3$)^{32}$. Drane subrayó que existen grados progresivos de exigencia. En su "Escala Móvil de Competencia" la anoréctica debe exhibir estándares más elevados de idoneidad y comprensión intelectual-emocional mientras más potencialmente delicada sea su elección (ej: rehusar alimentación) ${ }^{33}$. En cualquier caso, el diagnóstico de la enfermedad no es igual a diagnóstico de incapacidad, porque la anoréctica es perfectamente competente para tomar decisiones en otros campos de su vida.

3. Se necesita distinguir entre "rechazo" y "resistencia" al tratamiento. La anoréctica permanentemente se debate en una contradicción intema reprimida -necesidad de ser ayudada y su

Tabla 3. C apacidad para otorgar un consentimiento informado*

1. Susceptibilidad de ser informado

2. Capacidades cognitivas y afectivas

3. Toma de decisión

4. Revisión crítica del proceso de decisión

*Adaptado de: White BC Competence to consent. Washington: Georgetown University Press, 1994 32. 
aspiración a un ideal de belleza. La relación médica, al acompañar, hace posible que este rechazo inicial se modifique y exprese sólo como resistencia, que es un primer paso en la toma de conciencia de las verdades ocultas que generan su conflictiva -ansiedades persecutorias, aniquilación, exhibicionismo y culpa. Si después accede a iniciar una psicoterapia formal, comenzará su curación al interpretársele sus rígidas defensas y trasformará los rechazos voluntariosos y testarudos en opciones éticas de veracidad iluminación y trasparencia- para consigo ${ }^{34}$.

4. La decisión de sustitución se toma cuando la anoréctica está incapacitada temporal o permanentemente. Resulta complejísimo elegir por ella con un criterio justo y que la represente fielmente en sus valores íntimos, porque previamente se han tenido substanciales desaveniencias con ella. Se acostumbra dejarlo en manos de parientes más cercanos, aunque es recomendable seguir los estándares de mayor beneficio o del mejor interés, objetivamente medidos.

5. No basta suprimir síntomas o salvar la vida, es necesario ofrecer mejor calidad de vida. La calidad es un valor y no un hecho: la realización de valores que son preferidos a otros, resultado de elecciones íntimas de existencia. Contraniamente a la anoréctica, el médico valora tanto la vida -beneficencia- como su calidad, el "cómo". De ahí que en oportunidades se vea forzado a escoger no sólo entre medios proporcionados y medios desproponcionados de terapia, sino acudir al criterio de la excepcionalidad: definir en qué consiste la calidad mínima en determinadas circunstancias concretas y en vista de ciertas consecuencias precisas. Aunque puede entrar en conflicto con la autonomía, él precisa rebasar lo ordinario -pero respetando estrictamente la virtud de la "prudencia" y la noción de "persona razonable"- para conseguir una calidad intrínsecamente valiosa. La anoréctica podrá apreciar el resultado recién pasado el descalabro orgánico porque, se sabe experimentalmente, la emaciación origina una modificación del cerebro que, de manera indirecta, induce una alteración de su funcionamiento neurocognitivo estimativo ${ }^{2,15}$.

6. Con el tiempo los equipos terapéuticos suelen sucumbir al síndrome de burnout, desgaste profesional producto de la sensación de ser desbordados por las exigencias insaciables y contradictorias de las anorécticas. El médico necesita cuidarse del agotamiento emocional cumpliendo las recomendaciones éticas del "profesionalismo", pero especialmente analizándose y conociéndose a sí mismo -según el adagio, medice, cura te ipsum ${ }^{34-36}$. Además, al adoptar un modelo "deliberativo" de profesión - a diferencia de los modelos "absolutista" y "liberal"-, él tiene autoridad para cuestionar activamente valores y defender enérgicamente las razones de sus propias preferencias ${ }^{24}$.

7. Veredictos legales, cuyos dictámenes han sido históricos, han sometido al poder de la justicia parte significativa del proceder médico frente a la anoréctica. La oposición entre el orden ético y orden legal obliga al terapeuta a acatar los mandatos jurídicos, aun contradiciendo las guías de procedimiento terapéutico o sus convicciones íntimas. Es como si zonas sustantivas de la bioética de la anoréctica hubieran quedado sin moral y entregadas al arbitrio de los dictámenes punitivos de la autoridad judicial que, siguiendo las prácticas norteamericanas, sientan jurisprudencia y así, con cada sentencia, se profundiza la corrosión de los supuestos valóricos del accionar terapéutico.

\section{ÉTICA DE LA CONVICCIÓN Y ÉTICA DE LA RESPONSABILDAD}

La anoréctica parte del absoluto moral que sus ideales y la realización de éstos existen siempre e incondicionadamente, porque tienen primacía a priori sobre la realidad. Para ella, la indignidad proviene de la traición a estas convicciones imperativas y unívocas; si recurre a negociaciones, pactos o compromisos circunstanciales con los médicos, siente que se autoengaña, degrada o se aferra a los medios en lugar de perseguir los fines perfectos de su autorrealización.

También la medicina ha conocido como directriz de su quehacer esta moral de la excelencia, propia del "médico perfecto" u "óptimo", que se guía sólo por virtudes antes que por los efectos o resultados ${ }^{15}$. Empero, la anorexia constituye un ejemplo paradigmático del giro forzado que la medicina se vio obligada a efectuar durante el siglo XX hacia una ética de la responsabilidad.

Por lo analizado hasta aquí resulta claro que las consecuencias de los actos de la anoréctica son tan 
graves que no se pueden ignorar, por lo que el problema moral surge por conflictos de responsabilidad tanto o más que por principios en competencia (autonomía y beneficencia). Un atributo antropológico esencial sustenta esta afirmación: en bioética no es posible deducir reglas incondicionales de conducta desde principios morales universales. Vale decir, la razón práctica o valónica no proponciona certeza sino sólo probabilidad. Así, en el hombre no se puede hacer nunca exactamente lo que se debe, es el moral gap o fracaso moral inherente a la existencia $^{37}$. Fue Weber el que asumió esta insuficiencia radical de la razón y distinguió entre ética de la convicción (Gesinnungsethik) y de la responsabilidad para diferenciar entre la moral absoluta, propia de las intenciones internas, de la moral proporcional de las consecuencias, que toma en consideración las circunstancias y los efectos del obrar. La ética de la convicción basa su racionalidad en la estimación de los valores; la ética de la responsabilidad en el análisis del binomio medios-fines ${ }^{38}$.

En cierto sentido, ambas éticas son irreconciliables. La ética de la convicción es unilateral, no se detiene nunca, no encuentra jamás límites, ignora lo previsible, puede aun tornarse siniestra con el abuso, fanatismo e intransigencia. La ética de la responsabilidad está dirigida al futuro, considera lo que está por venir, lo imprevisto o el azar que no se pueden eliminar por completo. Solidez interior del "médico perfecto" frente a sensatez razonable del "médico prudente".

Pero indudablemente son complementarias. El terapeuta precisa armonizar la dialéctica mediosfines. Se actúa responsablemente cuando se proponen fines racionales y reflexivos, y se buscan los medios proporcionados para la consecución de esos fines. Eso significa tener simultáneamente a la vista las circunstancias concretas y considerar las consecuencias previsibles. En la anorexia no hay responsabilidad sin convicciones, así como toda convicción lo es en verdad cuando la prosecución de la meta se lleva a cabo con responsabilidad máxima hacia los desenlaces.

Para ser efectiva, esta responsabilidad necesita ser tanto compartida como deliberativa. Esto es, ambos participantes deben acatar dos condiciones básicas implícitas que, cuando no se respetan, comprometen severamente la interacción y la viabilidad de una terapia honesta. Por un lado, considerarse recíprocamente como interlocutores igualmente facultados o válidos y, por otro, que tienen algo en común, como es trabajar cooperativamente para comunicarse y comprenderse, aunque existan desacuerdos de principio ${ }^{39}$.

En otros términos, se es responsable con la anoréctica cuando: 1) se la respeta como ser humano individual; 2) las aspiraciones que se persiguen son reflexivas y sensatas; 3) la evaluación surge después de la ponderación de todos los factores concurrentes y consecuencias; 4) las decisiones se toman en forma deliberativa con ella; y 5) siempre se está abierto a la revisión de las conclusiones, aun ante las aparentemente más inconmovibles.

Hay que evitar dos malos entendidos procedentes de la utilización instrumental de la anoréctica para beneficio de las necesidades del terapeuta -nobles o personalistas-, esto es, la conversión de medios en fines; en expresión de Kant, trastocar la dignidad en precio ${ }^{29}$. En el primero, la táctica del médico consiste en aplicar una "racionalidad calculadora o tecnológica", que se limita a criterios técnicos que descansan sobre el saber empíico; el encuentro terapéutico es sólo una herramienta que acata reglas probadas, calcula los procedimientos limitándose a los datos observables, y mide el resultado calificándolo de verdadero o falso. En el segundo, el diálogo "estratégico" es el que emplea las reglas de elección racional o teoría del juego; tanto la anoréctica como el médico son considerados contrincantes racionales, toda acción se programa conjeturando las decisiones futuras de cada uno y los problemas de competición o cooperación se resuelven según las metas inmediatas del terapeuta -obtener consensos tácticos que aparentan un acuerdo, pero lo que pretenden es sortear obstáculos o resistencias para conquistar el triunfo 26,39 .

\section{¿Y EL CUIDADO?}

$\mathrm{Al}$ enfrentar los dilemas de la anoréctica hemos transitado desde una ética basada en "principios" equitativos o "convicciones" absolutas hacia una sustentada en "responsabilidades". Sin embargo, si queremos justificar nuestra elección es ineludible considerar las necesidades, entregar cuidados, proteger del desamparo, proporcionar apoyo, prevenir desequilibrios. Esto es, reconocer que en la anoréctica surgen conflictos importantes especialmente a causa de disputas entre responsabilidades, pero 
responsabilidades que hasta aquí no se han valorado suficientemente porque conciemen a atributos propios de las relaciones íntimas, como la compasión, fidelidad, empatía, solidaridad, honestidad, no-abandono. Por ello hay que recurrir a otra ética, una que posea un carácter relacional y concentrada en la reciprocidad entre médico-paciente.

La "ética del cuidado" está abocada al vínculo o attachment. El término cuidado apunta a la preocupación por el compromiso y la aspiración a jugárselas en beneficio de las personas con las que se tiene una relación estrecha o íntima. Lo importante para esta ética no es sólo lo que hace el profesional, sino cómo realiza sus actos, los motivos que los justifican y si favorece o no las relaciones positivas. Pero los motivos no pretenden constituir razones abstractas para conducir las acciones terapéuticas, sino intentan revelar los sentimientos, potencialidades, fortalezas, insuficiencias, carencias y sentido de la persona.

Arriba dijimos que la anorexia es una afección CEPA que, aunque tiene tratamiento potencialmente eficaz, es propia de la medicina abocada a los cuidados paliativos. Así, no se trata ni de una enfermedad aguda de tratamiento poderoso en que se conquista un éxito estrepitoso, ni de defender ideales utópicos, ni alcanzar las metas por encima de cualquier consideración -posición profesional, halago personal, ejecución virtuosa de una técnica. Frente al distanciamiento intelectual, pericia técnica o ideología combativa, importa la cercanía, empatía e intimidad personal inherente al cuidado. Es interrelación, negociaciones estrechas y cálidas, en las que ambos aspiran a entregar y recibir confirmación y apoyo y, de esta manera, conquistar un consenso confiado 40 . Reiterando, modelo "deliberativo" de profesión antes que "absolutista" y "liberal".

\section{REFERENCIAS}

1. Maj M, Halmi K, López-Ibor JJ, Sartorius N, eds. Eating Disorders. London: John Wiley \& Sons. 2003

2. Practice Guideline for the treatment of patients with eating disorders. $3^{\text {rd }}$ edition. Am J Psychiatry 2006; 163: 7 Supplement.

3. FAIRBURN CG. Evidence-based treatment of anorexia nervosa. Int J Eat Disord 2005; 37: 526-30.

4. National Collaborating Centre for Mental Health Eating Disorders. Core interventions in the treat-

\section{CONCLUSIONES}

1. Los principios de autonomía-beneficencia son los fundamentales al defender la libertad y la vida. Su jerarquía (relativa) hace indispensable que el terapeuta deba justificar con razones poderosas cuando se inclina por la beneficencia en contra de las preferencias de la anoréctica.

2. Las consecuencias graves $u$ ominosas hacen ineludible complementar la deliberación entre principios, con una ética de la responsabilidad que integre armoniosamente las convicciones individuales, los principios prima facie y la ponderación de los efectos.

3. Se aspira a acuerdos prudentes, razonables, deliberativos, revisables en el futuro y que respeten irrestrictamente a la anoréctica.

4. Al recordar que la anorexia requiere cuidados paliativos, la ética del cuidado presta atención a una arista moral hasta ahora desatendida: al vínculo, la vertiente relacional, comunicacional y de reciprocidad de la responsabilidad. Así los sentimientos, confianza, apoyo, compromiso, intimidad, precisan conquistar un puesto más preponderante para así ayudar a resolver mejor los dilemas morales.

5. Una ética adecuada a la anoréctica, que resuelva los deberes y obligaciones clínicas, significa armonizar dos órdenes morales médicamente heterogéneos: cuidado-proximidad-confianza y equidad-principios-ideales-obligaciones. Además de lo complejo de su elaboración teórica, requiere por parte del equipo de salud de una educación distinta a la recibida, que le permita deliberar y justificar de manera diferente los conflictos de valores.

ment and management of anorexia nerviosa, bulimia nervosa and related eating disorders. London: National Institute for Clinical Excellence, 2004.

5. Beauchamp TL, Childress JF. Principles of Biomedical Ethics. $5^{\text {th }}$ edition.New York: Oxford University Press, 2001.

6. SANTANDER F, coordinador. Ética y praxis psiquiátrica. Madrid: Asociación Española de Neuropsiquiatría, 2000.

7. Jonsen AR, Siegler M, Winsiade WJ. Clinical ethics. A practical approach to ethical decisions in clinical medicine. $5^{\text {th }}$ edition. New York: McGraw-Hill, 2002. 
8. Peliegrino ED, Thomasma DC. For the patient's good: The restoration of beneficence in health care. New York: Oxford University Press, 1988.

9. Watson T, BOWERS W, ANDERSON A. Involuntary Treatment of Eating Disorders. Am J Psychiatry 2000; 157: 1806-10.

10. Griffiths R, RusSell J. Compulsory treatment of anorexia nervosa patients. En: Vandereychken W, Beumont P, eds. Treating Eating Disorders: Ethical, Legal, and Personal Issues. London: The Athlone Press, 1998; p. 83-101.

11. Hebert PC, Weingarten MA. The ethics of forced feeding in anorexia nervosa. Can Med Assoc J 1991; 144: 145-51.

12. SilbER TJ. Justified paternalism in adolescent health care: cases of anorexia nervosa and substance abuse. J Adoles Health Care 1989; 10: 449-53.

13. Levi BH. Respecting patient autonomy. Urbana: University of Illinois Press, 1999.

14. Hope T, Savalescu J, Hendrick J. Medical ethics and law. The core curriculum. Edinburg: Churchill Livingstone, 2003.

15. Gracia D. Fundamentos de bioética. Madrid: Eudema, 1989.

16. WetTSTEIN RM. The right to refuse psychiatric treatment. Psych Clin North America 1999; 22: 173-81.

17. Mac Donald C. Treatment Resistance in Anorexia Nervosa and the Pervasiveness of Ethics in Clinical Decision Making. Can J Psychiatry 2002; 47: 267-70.

18. RATHNER G. A plea against compulsory treatment of anorexia nervosa patients. En: Vandereycken W, P. Beumont, eds. Treating Eating Disorders: Ethical, Legal, and Personal Issues. London: The Athlone Press, 1998; p. 45-57.

19. Vialettes B, Samuelan-Massat C, Valro R, Buard S. The refusal of treatment in anorexia nervosa, an ethical conflict with three characters: "the girl, the family and the medical profession". Diabetes Metab 2006; 32: 306-11.

20. Verkerk M. A Care Perspective on Coercion and Autonomy. Bioethics 1999; 13: 358-68.

21. Dunn LB, Nowrangi MA, Palmer BW, Jeste DV, Saks ER. Assessing decisional capacity for clinical research or treatment: A review of instruments. Am J Psychiatry 2006; 163: 1323-34.

22. Simón P. El consentimiento informado. Historia, teoría y práctica. Madrid: Triacastela, 2000.
23. McCormick RB. The quality of life. The sanctity of life. Hastings Center Report 1978; 8: 30-41.

24. Gracia D. Como arqueros al blanco. Estudios de bioética. Madrid: Triacastela, 2004.

25. Figueroa G. Responsabilidad profesional: máximos, mínimos, excelencia y veracidad. Rev Méd Chile 2006; 134: 251-7.

26. Cortina A. Ética del discurso y bioética. En: Blanco Fernández D, Pérez Tapias JA, Sáez Rueda L, eds. Discurso y realidad. En debate con K.O. Apel. Madrid: Trotta, 1994; p. 75-89.

27. ARISTóteles. Ética a Nicómaco. Madrid: Centro de Estudios Constitucionales, 1981.

28. MiL JS. On Liberty. Harmondsworth: Penguin Books, 1982.

29. KANT I. Grundlegung zur Metaphysik der Sitten En: Kant-Studienausgabe Band VI. Darmstadt: Wissenschaftliche Buchgesellschaft, 1968; p. 1-102.

30. Simón P, BARRIo IM. La capacidad de los menores para tomar decisiones sanitarias: un problema ético y jurídico. Rev Esp Pediat 1997; 53: 107-18.

31. JauRegui Lobera I. Trastomos de la conducta alimentaria. La madurez psicológica del menor para la toma de decisiones. Tratamiento forzado. Rev Psiquiatr Psicol Niño Adolesc 2002; 1: 23-32.

32. WhITE BC. Competence to consent. Washington: Georgetown University Press, 1994.

33. Drane J. The many faces of competency. Hastings Center Rep 1985; 15: 17-21.

34. Figueroa G. La bioética en el diván. ¿Puede Freud ayudarnos en los dilemas de la ética médica? Rev Méd Chile 2004; 132; 1550-6.

35. FIgUEROA G. Bioética y psicoterapia. ¿Cuáles supuestos morales actúan cuando ejecutamos un acto psicoterapéutico? Rev Méd Chile 2004; 132: 243-52.

36. Gracia D. Medice, cura te ipsum. Sobre la salud física y mental de los profesionales sanitario. Madrid: Instituto de España y Real Academia Nacional de Medicina, 2004

37. HARE JE. The moral gap: Kantian ethics, human limits, and god's assistance. Oxford: Clarendon Press, 1996.

38. WeBer M. Politik als Beruf, Wissenschaft als Beruf. Berlin: Dunker \& Humboldt, 1959.

39. Apel K-O. Diskurs und Verantwortung. Frankfurt: Suhrkamp, 1988.

40. Have HT, Clarck D, eds. The ethics of palliative care: European perspectives. Buckingham-Philadelphia: Open University Press, 2002. 